\title{
Norois
}

Environnement, aménagement, société

$235 \mid 2015$

La directive cadre sur l'eau à l'échéance 2015. Les

façades océaniques

\section{Un « livre blanc » sur la géographie}

\section{Abdelkader Mohaine}

\section{(2) OpenEdition}

Journals

Édition électronique

URL : http://journals.openedition.org/norois/5661

DOI : $10.4000 /$ norois.5661

ISBN : 978-2-7535-4775-9

ISSN : $1760-8546$

\section{Éditeur}

Presses universitaires de Rennes

\section{Édition imprimée}

Date de publication : 15 novembre 2015

Pagination : 154-156

ISBN : 978-2-7535-4771-1

ISSN : 0029-182X

\section{Référence électronique}

Abdelkader Mohaine, « Un « livre blanc » sur la géographie », Norois [En ligne], 235 | 2015, mis en ligne le 15 novembre 2015, consulté le 23 septembre 2020. URL : http://journals.openedition.org/norois/ 5661 ; DOI : https://doi.org/10.4000/norois.5661

\section{(c) Tous droits réservés}


l'agriculture péri-urbaine étant, en France au moins, une préoccupation importante dans le contexte d'étalement urbain qu'elle connaît.

Dans ce contexte de difficile partage de l'espace, ce n'est pas un hasard si l'un des chapitres propose une analyse du foncier. C'est en effet l'un des principaux freins au maintien de l'agriculture et à la protection de la nature, « avec la spécificité de la problématique foncière sur le littoral », comme le soulignent Clotilde Buhot et Yann Gérard dans leur introduction intitulée «Étudier le foncier, un enjeu pour l'aménagement des littoraux méditerranéens ».

Finalement, les deux premiers chapitres « Les limites du littoral » et « L'urbanisation du littoral » sont, a priori, les moins novateurs car ils reprennent des éléments de connaissance déjà analysés sur d'autres littoraux et sur lesquels de très nombreuses publications ont été réalisées. Cependant, chacun est agrémenté d'études de cas qui apportent des éclairages pertinents sur ces thématiques et le troisième chapitre intitulé «Conflits sur le littoral » vient à point nommé, soulignant la crispation des jeux d'acteurs résultant d'une difficulté à organiser l'espace littoral.

Deux éléments sont à regretter dans cet ouvrage. Tout d'abord, les exemples portent, à l'exception d'une contribution sur le Grand Tunis, sur la partie nord de la Méditerranée. Il aurait été intéressant d'illustrer «L'avenir de la méditerranée face à l'urbanisation » par d'autres portions du littoral méditerranéen. Ensuite, il est dommage que l'ouvrage s'appuie sur des constats et ne répond que très peu à la question qu'il pose « Un littoral sans nature? » et qu'au final, peu d'études de cas permettent de dessiner l'avenir de ce littoral.

Il n'en reste pas moins que l'ouvrage coordonné par Coline Perrin et notamment les études de cas qu'il contient, constitue un apport intéressant pour qui se préoccupe des questions littorales.

Céline Chadenas Université de Nantes

André Humbert, 2012. Le géographe et le tapis volant, Madrid, La Casa de Velázquez, «Coll. de la Casa de Velázquez; 5 », ISBN 9788496820869, 206 p.

Un «livre blanc» sur la géographie. Ce qualificatif ne serait sans doute pas exagéré pour désigner l'ouvrage, dans la mesure où celui-ci revient, indirectement il est vrai, sur la nature véritable de la géographie, comme si l'auteur voulait mettre en garde contre les errances dans lesquelles l'ont entrainé certains géographes de la nouvelle génération. Le livre n'est certes pas «un exercice académique» (p. XIII), mais c'est précisément «cette réflexion libre » (p. XI) qui lui a permis d'apporter beaucoup d'éléments objectifs et factuels. Le livre, qui est l'aboutissement de 40 années d'une aventure peu commune parmi les corporations des géographes, est organisé en deux parties : l'une aéronautique et l'autre géographique.

Durant toute la partie aéronautique, l'auteur embarque avec lui le lecteur en lui faisant vivre toutes les sensations du vol artificiel tantôt marqué par des moments de stress et d'angoisses, d'où une «inquiétude permanente» (p. 15) et la hantise de la chute, et tantôt par des moments d'exaltation

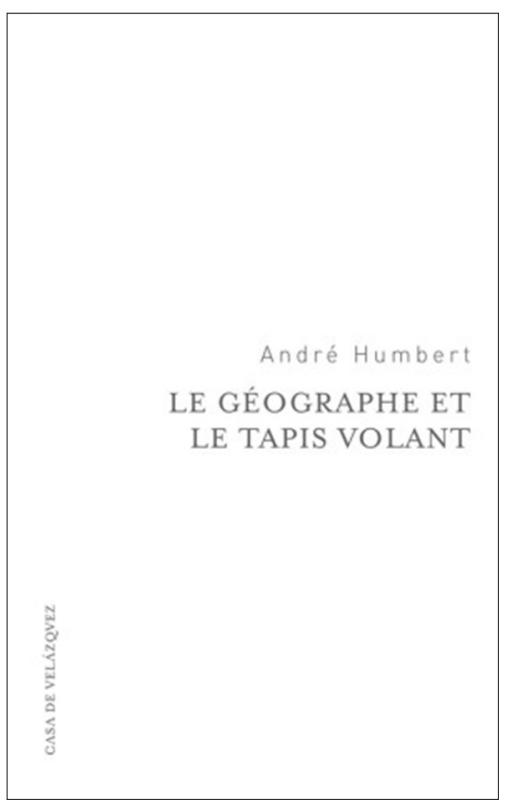


marqués par un «sentiment de délivrance [...], de jubilation [...et] de béatitude» (p. 3). C'est aussi durant ces moments que l'auteur bénéficie d'un recul altimétrique sans précédent et que le métier du géographe qu'il est prend le dessus sur la passion du pilote qui l'anime. En même temps il observe, décrit - dans un style parfois lyrique - analyse et immortalise les paysages qui s'offrent à lui. Rien, ou presque, ne lui échappe à tel point qu'il est difficile de savoir si l'auteur volait pour être géographe ou s'il était géographe pour voler. Ce qui est sûr, en revanche, c'est qu'il a réussi à associer « le plaisir du vol à [son] métier de géographe» (p. 7).

Avec des exemples concrets pris au Maroc, en France et dans la péninsule ibérique, l'auteur, avec son talent de géographe, laisse libre cours à toute son imagination. Il illustre les tableaux paysagers qu'il découvre : vues d'avion les montagnes et les côtes marines prennent d'autres dimensions, et les paysages façonnés par l'homme deviennent des œuvres d'art qui témoignent indéniablement de son degré de civilisation. En un mot emprunté à Antoine de Saint-Exupéry (Terre des Hommes), l'auteur n’ «admire [plus les paysages] mais [il] les médite».

Après avoir rappelé l'utilité de l'étude des paysages en insistant sur cet « handicap majeur » (p. 57) dont souffre le géographe qui n’a pas la possibilité de regarder son objet d'étude dans son ensemble, l'auteur se livre à une véritable leçon de géographie générale à laquelle il consacre toute la deuxième partie (deux tiers de l'ouvrage). Et c'est par ce véritable laboratoire de géographie rurale - ou musée vivant - qu'est l'Anti Atlas marocain et certaines de ses bordures qu'il a inauguré cette partie géographique. Il a d'abord décrit et essayé d'expliquer comment les habitants de cette «terre des hommes» (p. 65) ont su s'accrocher, « le mot n'est pas trop fort » (p. 65) appose t-il, à un milieu aussi difficile, voire parfois inhospitalier.

L'auteur analyse le fonctionnement, dans le temps et dans l'espace, des stratégies d'adaptation des populations de ces contrées largement défavorisées par la nature et passe en revue les techniques - ingénieuses - de gestion d'une économie de rareté, tout en rappelant le savoir-faire d'une communauté solidaire et stoïque, mais privée de plus en plus de la main d'œuvre mâle et jeune que lui a soustraite l'émigration. Les systèmes d'irrigation et ceux des cultures sont soigneusement décor- tiqués. L'organisation des territoires montagneux n'en demeurent pas moins analysés. L'habitat de la collectivité traditionnelle de l'Anti Atlas et ses techniques ancestrales d'emmagasinage (les Agadir-s ou les Igoudar) sont clairement décrits et expliqués. Afin de mieux faire ressortir les particularités de cette montagne, l'auteur la compare dans un premier temps avec la puissante chaine du Haut Atlas et ensuite avec le désert - si proche - où la vie en ilots est permise grâce à la stratégie (technique) de captage et de drainage des eaux souterraines (les Khetteras). Mais les impacts de la concurrence sur l'eau, nous dit l'auteur, livrée essentiellement par une agriculture ultra moderne des plaines et l'effritement de la communauté, commencent à se faire sentir sur ces installations collectives plus que millénaires.

Ce sous chapitre consacré à l'Anti Atlas est riche en vocabulaire géographique local dont l'auteur maitrise parfaitement le sens. Il en donne même, parfois, des équivalents en espagnol ou en français. Il ne manque pas non plus de s'interroger sur certains concepts et d'en proposer d'autres, faute de terme générique, souligne t-il.

Les milliers de kilomètres de côtes survolées entre Dunkerque et Sidi Ifni ou encore entre l'Italie et Gibraltar ont fait l'objet d'une longue synthèse « comparative » de paysages littoraux très divers et différemment occupés. Le relief, les marais, les salins, les rizières, les ports de pêche, les ports industriels et les installations touristiques ont fait l'objet d'une véritable étude de géographie des littoraux.

Une attention particulière est également accordée à « la polygénie urbaine maghrébine » (p. 108-117) notamment aux médinas mais aussi à un « exemple [...] de la ville marocaine moderne, sans histoire » (p. 116). Le recul altitudinal que lui a offert son tapis volant lui a permis à la fois de reposer le problème de l'orientation des édifices religieux ô combien important dans les pays musulmans et de rappeler indirectement le caractère "tronqué » des études microscopiques de géographie urbaine actuelle.

Il n'y a peut-être pas une ligne de ce livre qui ne se ressent des circonstances dans lesquelles il a été rédigé. Loin du style des ouvrages académiques, l'ouvrage est rédigé dans un style ciselé qui allie un langage très soutenu et un discours géographique (et aéronautique) pertinent. C'est un ouvrage qui fait 
redécouvrir la Géographie sous un angle peu connu, voire inédit, pour les géographes. Les champs disciplinaires auxquels l'auteur a fait appel sont aussi nombreux que variés, comme pour rappeler que la géographie est (encore et toujours) généraliste et que le géographe doit effectivement être " pilote », autrement dit, mettre les phénomènes étudiés dans leur contexte global.

Abdelkader MoHaine Géographe, Université d'Agadir

Philippe DE LARMinAT, 2014. Changement climatique : identification et projections, ISTE (Ed.), 138 p.

Dans son ouvrage l'auteur nous propose un nouveau regard sur la modélisation des processus du changement climatique : l'identification. La théorie de l'identification des systèmes dynamiques est éprouvée depuis plusieurs décennies mais principalement dans le domaine industriel. Or, l'auteur nous démontre qu'elle a toute sa place dans la modélisation climatique planétaire. Pourtant, aucun article scientifique n’y fait référence!

En quoi consiste l'identification et qu'est-ce que cette méthode apporte de nouveau? Il s'agit de déterminer un modèle mathématique à partir des observations en entrée et en sortie du système que l'on souhaite étudier. En d'autres termes, trouver (identifier) les liens entre les causes et les effets. Dans le sujet qui nous intéresse, les entrées sont la concentration du dioxyde de carbone atmosphérique, l'activité solaire ou encore l'activité volcanique. La sortie est la température de surface du globe. On parle ici de modèle «boite noire ", c'est-à-dire que les liens entre les entrées et la sortie sont purement mathématiques, à aucun moment cette modélisation ne cherche à représenter physiquement la réalité des processus mis en œuvre. La méthode est donc « libre » et non contrainte a priori.

Les modèles du GIEC $^{1}$ sont tout le contraire : ce sont des modèles numériques basés sur les lois physiques. Ainsi les résultats obtenus par ces modèles dépendent fortement des lois implémentées. Ces modèles sont donc d'une part fortement contraints par les variables et paramètres des équations qui les construisent et d'autre part évidemment incapables

1. Climate Change 2013. The Physical Science Basis: Working Group I Contribution to the Fifth Assessment Report of the Intergovernmental Panel on Climate Change. (Cambridge University Press, 2014). [http:// ebooks.cambridge.org/ref/id/CBO9781107415324].

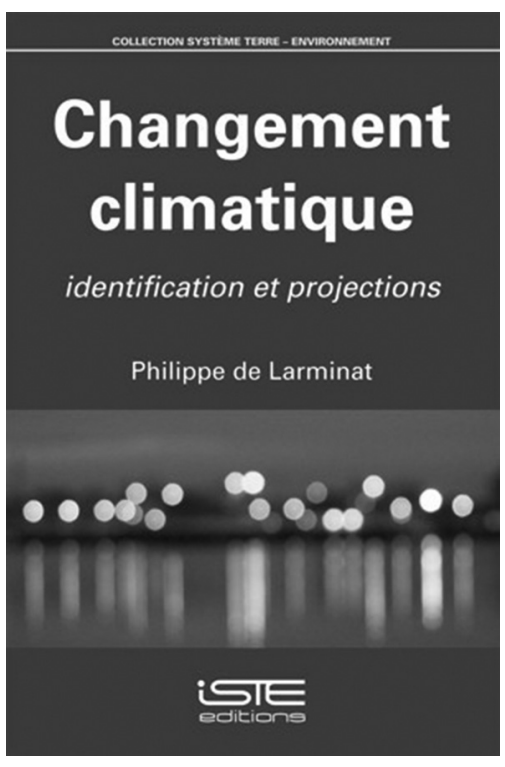

de reproduire des processus qui n'ont pas été pris en compte parce que, par exemple, trop complexes.

La posture de l'auteur dans tout l'ouvrage est clairement tournée vers le partage scientifique et la confrontation constructive des idées. Il présente de façon partiale les données climatiques produites par les grands instituts (e.g. National Oceanic and Atmospheric Administration, Climate Research Unit), et indique que les modèles qu'il a appliqués sur ces données peuvent être téléchargés sur le site de l'éditeur afin que chacun puisse reproduire ses expériences in silico. L'auteur nous rappelle la guerre provoquée par ces données et leurs représentations graphiques (la courbe en crosse de hockey de Mann ${ }^{2}$ par exemple). On découvre également le

\footnotetext{
2. Mann M. E., Bradley R. S. \& Hughes M. K., 1998. Global-scale temperature patterns and climate forcing over the past six centuries. Nature, 392, p. 779-787.
} 